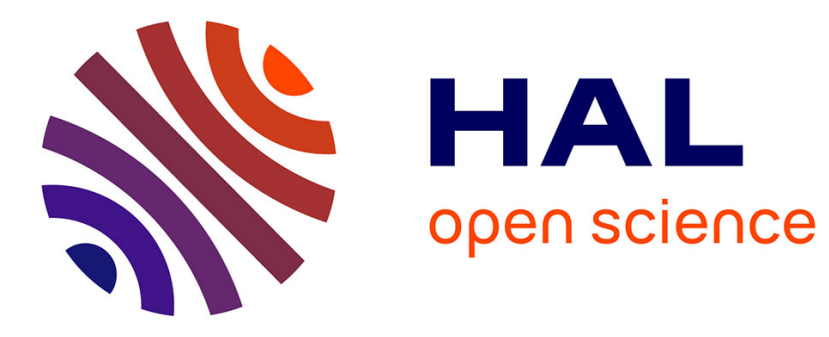

\title{
VASAO: visible all sky adaptive optics
}

Christian Veillet, Olivier Lai, Derrick Salmon, J. P. Pique

\section{To cite this version:}

Christian Veillet, Olivier Lai, Derrick Salmon, J. P. Pique. VASAO: visible all sky adaptive optics. Proceedings of SPIE, the International Society for Optical Engineering, 2006, Advances in Adaptive Optics II. Edited by Ellerbroek, Brent L.; Bonaccini Calia, Domenico., 6272, pp.637221. $10.1117 / 12.672750$. hal-01118414

\section{HAL Id: hal-01118414 https://hal.science/hal-01118414}

Submitted on 3 Mar 2015

HAL is a multi-disciplinary open access archive for the deposit and dissemination of scientific research documents, whether they are published or not. The documents may come from teaching and research institutions in France or abroad, or from public or private research centers.
L'archive ouverte pluridisciplinaire HAL, est destinée au dépôt et à la diffusion de documents scientifiques de niveau recherche, publiés ou non, émanant des établissements d'enseignement et de recherche français ou étrangers, des laboratoires publics ou privés. 


\title{
VASAO: Visible All Sky Adaptive Optics
}

\author{
Christian Veillet $^{* a}$, Olivier Lai ${ }^{a}$, Derrick Salmon ${ }^{\mathrm{a}}$, Jean-Paul Pique ${ }^{\mathrm{b}}$ \\ ${ }^{a}$ Canada France Hawaii Telescope, 65-1238 Mamalahoa Hwy., Kamuela HI 96743, USA ; \\ ${ }^{\mathrm{b}}$ Laboratoire de Spectrométrie Physique, Université Joseph Fourier, UMR 5588 CNRS- \\ Grenoble I, B.P. 87, 38402 Saint Martin d'Hères, France
}

\begin{abstract}
Building on an extensive and successful experience in Adaptive Optics (AO) and on recent developments made in its funding nations, the Canada-France-Hawaii-Telescope Corporation (CFHT) is studying the VASAO concept: an integrated AO system that would allow diffraction limited imaging of the whole sky in the visible as well as in the infrared. At the core of VASAO, Pueo-Hou (the new Pueo) is built on Pueo, the current CFHT AO bonnette. Pueo will be refurbished and improved to be able to image the isoplanetic field at $700 \mathrm{~nm}$ with Strehl ratios of $30 \%$ or better, making possible imaging with a resolution of 50 milliarcseconds between 500 and $700 \mathrm{~nm}$, and at the telescope limit of diffraction above. The polychromatic tip-tilt laser guide star currently envisioned will be generated by a single $330 \mathrm{~nm}$ mode-less laser, and the relative position of the $330 \mathrm{~nm}$ and $589 \mathrm{~nm}$ artificial stars created on the mesosphere by the $330 \mathrm{~nm}$ excitation of the sodium layer will be monitored to provide the atmospheric tip-tilt along the line of sight, following the philosophy developed for the ELP-OA project. The feasibility study of VASAO will take most of 2006 in parallel with the development of a science case making the best possible use of the unique capabilities of the system, If the feasibility study is encouraging, VASAO development could start in 2007 for a full deployment on the sky by 20112012.
\end{abstract}

Keywords: Adaptive optics, Laser guide star, Polychromatic, Visible, All-sky

\section{A BRIEF HISTORY OF IMAGE QUALITY IMPROVEMENT AT CFHT}

Canada France Hawaii Telescope (CFHT) has been known since its early days for its image quality, starting in the early 80 s with wide-field imaging, thanks to large photographic plates and a good corrector at its prime focus. The arrival of CCDs in the mid-80s caused the demise of photography, even though the size of the detectors was small and field of view therefore limited. By the end of the 80 s, a new CCD camera, HRCAM, was installed at prime focus, providing realtime tip-tilt corrections to improve the image quality by reducing the degradation due to telescope tracking errors and image motion to atmospheric (seeing) effects ${ }^{1}$. The CFHT adaptive optics bonnette project (AOB, or Pueo) started back in 1991. It uses curvature sensing, a concept applied to adaptive optics (AO) by Francois Roddier ${ }^{2}$. It features a 19 elements bimorph mirror and curvature sensor, and was largely inspired by the AO system developed at the Institute for Astronomy (University of Hawaii) by the UHAO group. Pueo ${ }^{3,4,5}$ arrived at CFHT in January 1996. Engineering and commissioning tests on the telescope were performed during the first semester of 1996 and Pueo has since then been offered to the CFHT users.

For its ease of use, reliability and performance, Pueo has been one of the best AO systems in operation on a telescope, making the best of the excellent natural seeing on Mauna-Kea by routinely providing diffraction images in the infrared. Pueo's operation has been a model for subsequent systems developed on 8 to $10-\mathrm{m}$ telescopes. Since the general availability of adaptive optics on larger telescopes like Gemini or the VLT, Pueo lost some of its appeal and therefore the pressure on Pueo decreased slowly but surely. Meanwhile, with the development of mosaics of large size CCDs, widefield astronomy was again possible at CFHT and $\mathrm{CFH} 12 \mathrm{~K}^{6}$, hosted in the old prime focus cage used in the early days for large photographic plates, saw first light in early 1999 and became rapidly a heavily used instrument for highly ranked programs. It was replaced in 2003 by $\mathrm{MegaCam}^{7}$, the largest digital camera currently in operation on a telescope with

*veillet@cfht.hawaii.edu phone 1808 885-7944; fax 1808 885-7288; cfht.hawaii.edu 
340 MegaPixels, complemented by the end of 2005 with WIRCam ${ }^{8}$, a 4k x 4k infrared camera. MegaCam, offering 0.18 arc-second pixels over a 1 square degree field, offers a relatively high angular resolution but is essentially seeing limited.

Visible and infrared wide-field imaging with good image quality and well-matched spatial sampling is currently the niche of the telescope. MegaCam and WIRCam are under heavy demand, leading to oversubscription on CFHT's observing time as high as 3 to 4 in Canada and France. This is likely to continue up to the end of the decade. However, these cameras will ultimately lose some of their interest with the development of larger cameras, both in the visible and the infrared, on dedicated telescopes. The image quality and sampling could still be among the best available. However, for programs where sky coverage is more important than spatial resolution, CFHT will not be as competitive as today. It is therefore time to look at an observational niche that would make good use of the Mauna Kea site and of the relatively modest size of CFHT's 3.6-m telescope in the horizon 2011-2012.

Building on the experience of Pueo, why not come back to adaptive optics, but this time in the visible and with wholesky coverage? VASAO, "Visible All-Sky Adaptive Optics", started to take shape in mid-2005 and was formalized as a real CFHT project entering a feasibility phase by the end of that year. VASAO is an option well tuned to CFHT on Mauna Kea: (1) AO highly benefits from good natural seeing. (2) The smaller the telescope, the easier diffraction limited imaging is in the visible. (3) The elevation of the site provides a good transparency in the blue. (4) The 3.6-m CFHT is well-suited to $\mathrm{AO}$ in the visible with a single laser guide star. Unlike with the 8 to $10-\mathrm{m}$ class telescopes, the wave front error coming from the LGS cone effect, linearly depending on the diameter of the telescope, does not prevent reaching the diffraction limit of the telescope in the visible. In addition, whole-sky capabilities allow observing wherever there is something of interest, including places where other telescopes cannot observe, as even laser-assisted adaptive optics systems still need a natural star for tilt correction. Were VASAO to be in operation in 2011, CFHT would likely be the only ground-based telescope able to observe everywhere in the sky at the diffraction limit of the telescope.

\section{ELP-OA AND THE POLYCHROMATIC LASER GUIDE STAR}

The VASAO concept is making use of the pioneering work of many colleagues, which led to the realization that VASAO could indeed be feasible. The use of a polychromatic laser guide star is clearly the key to this (still to be demonstrated) feasibility. Twenty years after the first paper ${ }^{9}$ on the use of an artificial star for adaptive optics (AO) correction in absence of any bright source in the observed field, laser guide stars (LGS) AO systems are now in operation or in development on many telescopes, offering routinely diffraction limited images in the infrared at the Keck Observatory ${ }^{10}$. There is however one limitation in the classical LGS approach: the apparent direction of the LGS is independent of the atmospheric tilt and of the telescope vibrations, tracking errors or wind shakes. Therefore, a nearby star is still needed to complement the LGS AO system with the tilt information. Unfortunately, there is a good fraction of the sky, from relatively obscure star forming regions to fields far from the galactic plane, where there is no star close and bright enough to provide this essential tilt measurement. Foy and Migus ${ }^{11}$ proposed in 1995 the concept of the polychromatic artificial sodium star as a way to overcome this difficulty and to open the whole sky to AO observations.

Based on the Polychromatic LGS (PLGS), the ELP-OA ("Etoile Laser Polychromatique pour l'Optique Adaptative") project was initiated in France by R. Foy. ELP-OA's various steps and progresses have been extensively described in the literature $^{12,13}$. A more general study of the use of ELP-OA on telescopes of various sizes assuming various laser fluxes and launch telescope diameters has been carried out ${ }^{14}$, opening the road to the potential use of the concept for real operation.

The PLGS is created through the two-photon excitation of the sodium atoms at the sodium mesospheric layer as shown on Figure 1. The two artificial stars at 330 and $589 \mathrm{~nm}$ are created at the same spot of the mesosphere, but will be affected by the atmospheric tilt in a different way, leading to the measurement of the tilt variations through the variation of the apparent distance between the two stars.

The PLGS does not fully replace the natural tip-tilt star used in LGS-AO systems: it does not give any information on the telescope vibrations, tracking errors and wind shakes, which, nevertheless, have to be monitored. ELP-OA makes use of a pair of pendular seismometers first proposed by Tokovinin ${ }^{15}$ for this monitoring. Prototypes have been developed and shown to track the telescope vibrations to a few milliarcseconds. 


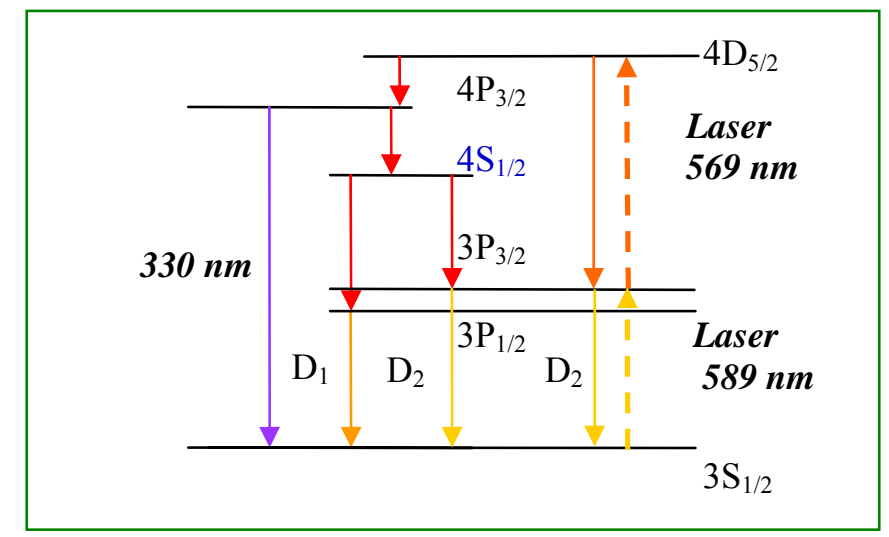

Fig.1 The two-photon excitation of the sodium atom. The $589 \mathrm{~nm}$ laser is producing the usual $\mathrm{D}_{2}$ excitation used for the AO correction on conventional LGS. The $569 \mathrm{~nm}$ laser will excite the already excited atoms up to $4 \mathrm{D}_{5 / 2}$, releasing photons at both $330 \mathrm{~nm}$ and $589 \mathrm{~nm}$.

ELP-OA is now moving to a demonstration phase at Observatoire de Haute Provence on the 1.52-m telescope. Though there will not be any adaptive optics system on this telescope, the goal is to close the tip-tilt control loop using the PLGS generated using the two-photon excitation illustrated in Figure 1 and the pendular seismometers, in order to evaluate the performances as a function of the laser flux, the seeing and the wavelength.

\section{VASAO IN SHORT}

\subsection{Specifications (or dreams at this stage!)}

The main characteristics of VASAO can be summarized in very general terms as follows:

- 50mas imaging in the visible (Total residual wave front error $\sim 120 \mathrm{~nm}$ rms leading to a Strehl of $22 \%$ at $500 \mathrm{~nm}$ and $>90 \%$ at $2.2 \mathrm{um}$ )

$-5 \%$ or less down time on the sky due to instrument problems and failures,

- operation by non-specialist,

- 1-day max system preparation time,

- duty cycle up to $80 \%$,

- 10-year life time,

- automated (or easy to use) system diagnosis (as much as possible!) from all main components,

- automated configuration,

- ultra-stable mechanical and optical configuration,

- on-axis laser projection.

Design should be done with the following constraints:

- minimize the development on Pueo-Hou,

- maintain dual capability: LGS or NGS for both tip-tilt and AO correction,

- minimize free air laser propagation,

- operate exclusively in queued service observing mode (QSO)

- complete the project by 2011/2012.

At this stage, some of the requirements are goals which will be hard to reach and the feasibility study will likely come to more realistic specifications. However, the strong goals on the development of the project and on the operation of VASAO once on the sky have immediate consequences on the design: learning from the experience of complex laser systems, robustness and simplicity will be a key to the success of the project. The specifications of VASAO are already very stringent, and it would not be reasonable to consider anything but a natural isoplanetic field of view; anything larger would require an investment well beyond the scope of VASAO. 


\subsection{How to phase VASAO development and ELP-OA?}

A careful analysis of the important steps (simulations, laboratory experiments or on-sky tests) accomplished so far by ELP-OA related to the operational requirements of VASAO led to a set of basic observations as follows:

- The two-photon excitation as already been demonstrated on the sky by the PASS-2 experiment ${ }^{16}$. While straightforward, this mode is not appealing from an operational perspective. (1) A dye laser has to be used (not as operation-friendly as a solid-state laser). (2) Two lasers have to be synchronized and launched to hit the mesosphere at the same place.

- The use of the differential motion of the components of different colors from the same star has also been demonstrated on the sky with the ATTILA experiment ${ }^{17}$.

- A mode-less laser has been developed and tested in the laboratory by J.P. Pique ${ }^{18}$. By exciting the whole velocity classes and hyperfine structure of the sodium line, such a laser is more efficient at generating a sodium laser guide star in the mesosphere than a single- or multi-mode laser.

- Prototypes of the Tokovinin's pendular seismometers have been built and proven to give the required accuracy in angular displacement monitoring, though their first design proved to be fragile. A new design is actually in the work within the ELP-OA project.

- The optimal diameter of the laser launch telescope at a site like Mauna Kea, related to the atmospheric coherence length in good seeing conditions, is going to be larger than at OHP due to a much better seeing.

With these observations in mind, it became clear that VASAO should go its own route, while sharing with the ELP-OA demonstration phase at OHP all the simulation, design and development information.

\section{VASAO MAIN COMPONENTS}

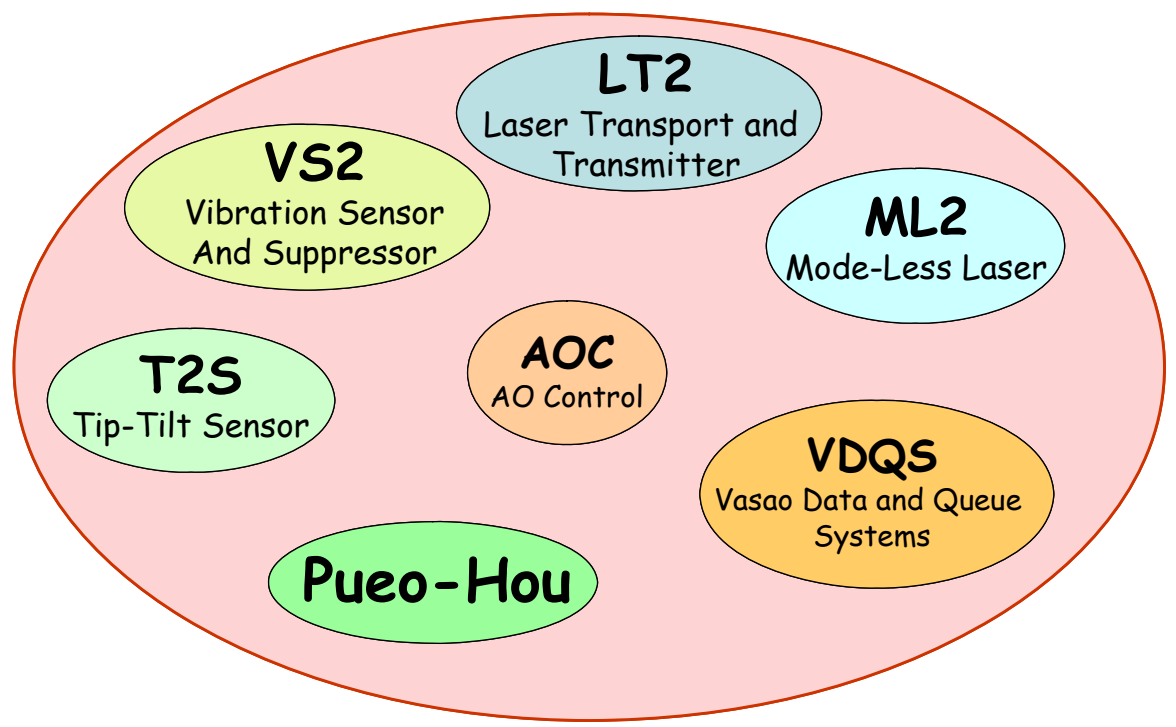

Fig.2. Block diagram of VASAO with its main functional components.

The main components of VASAO are shown on Figure 2.

\subsection{Pue Hou}

Pueo Hou (the "new" Pueo) is at the heart of VASAO. It is essentially an upgrade of the existing AOB, keeping its overall optical layout untouched, in particular the wave front sensor (WFS) component, in order to minimize the developments and the associated risks of building a new AO system. It will "only" require new coatings on many of the optical components to accommodate the $330 \mathrm{~nm}$ photons, a new deformable mirror and a new lenslet array, hopefully using as WFS detector the Fly-Eyes ${ }^{19}$ concept currently in development at CFHT and to be tested on the sky before the end of 2006. 


\subsection{ML2}

ML2, the Mode-Less Laser, will generate the polychromatic laser guide star. At this point, it is envisioned to work with a single laser at $330 \mathrm{~nm}$, using a scheme proposed by J.P. Pique ${ }^{20}$. The excitation of the mesospheric sodium is done as shown on Figure 3.

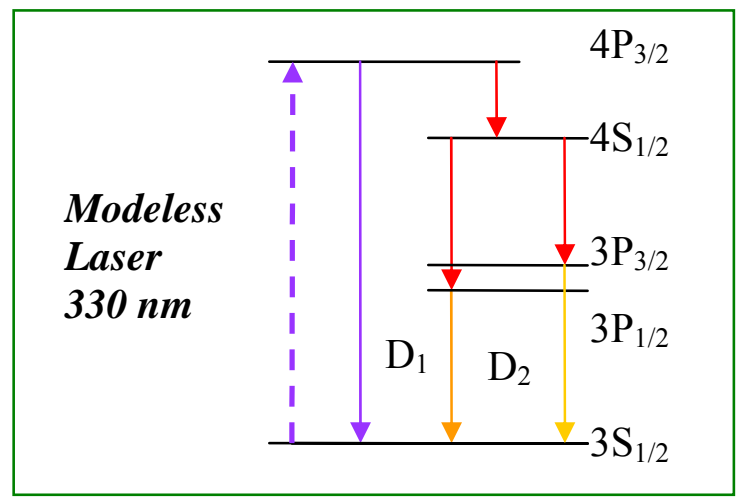

Fig. 3. The $330 \mathrm{~nm}$ excitation of the sodium atom. A single laser generates both $330 \mathrm{~nm}$ and $589 \mathrm{~nm}$ laser guide stars.

Compared to the two-photon excitation proposed for ELP-OA, the 330nm excitation offers the advantage of a single laser instead of two, which means less complexity and easier operation, avoiding the need for synchronizing and collocating to laser beams. A $330 \mathrm{~nm}$ beam can be generated by quadrupling the frequency of a Nd:YLF laser at $1321 \mathrm{~nm}$, allowing for a unique solid-state laser. If the flux levels are confirmed by further studies, ML2 would be used for the both the PLGS and the laser guide star used for the AO correction.

\subsection{LT2}

At this stage, the launch telescope will be $50 \mathrm{~cm}$ in diameter, a few times the average value of the Fried parameter $\mathrm{r}_{0}$. It should be mounted on the secondary mirror of the Cassegrain configuration of the telescope. Laser projection will therefore be centered, minimizing the elongation of the laser spot seen by Pueo Hou. It should be noted that a curvature system like Pueo Hou is not centroiding the laser spot, but only using its overall sub-pupil intensity; by design, it is therefore much less sensitive to this elongation that Shack-Hartman systems.

The transport of the laser at $660 \mathrm{~nm}$ will hopefully be done through hollow core fibers, minimizing free-air propagation and safety issues. The second SHG crystal will be placed at the fiber output to produce $330 \mathrm{~nm}$. It should be noted that a laser beam at $330 \mathrm{~nm}$, though not completely safe, is less dangerous than at $589 \mathrm{~nm}$, as the cornea is not transparent at $330 \mathrm{~nm}$.

\subsection{T2S}

T2S, the Tip-Tilt Sensor, is essentially made of an efficient low noise detector able to image the $330 \mathrm{~nm}$ and $589 \mathrm{~nm}$ images of the PLGS at the proper rate (a few tens of ms).

\subsection{V2S}

As already explained, the vibrations and wind shakes of the telescope have to be monitored. If the pendular seismometers can work flawlessly in operation, they should make for the ideal vibration sensor. The angular displacements of the telescope they will provide will be added to the atmospheric tilt information provided by the PLGS.

\subsection{AOC and VDQS}

The AO Control component will have to take care of the whole AO operations. As seen in the VASAO specifications section, the design goal is to build in the system as many automated tasks as possible, from setups through operation to diagnostics. VASAO will be used in Queued Service Observing like the wide-field imagers currently on the telescope for nearly 300 nights a year. However, by comparison, WIRCam and MegaCam are easy instruments to operate! Minimizing the staff around the telescope when VASAO is in use on the sky is one of the drivers of the design in this feasibility study. Real time data analysis and automated program selection as a function of the VASAO performances are some of the tasks the VASAO Data and Queue systems will have to handle. 


\section{SIMULATIONS IN PROGRESS - IDENTIFYING THE CHALLENGES}

\subsection{Flux from the laser}

In the various simulations made so far, we have used the estimates made by J.P. Pique for a $330 \mathrm{~nm}$ delivering $10 \mathrm{~W}$ at the mesosphere as shown on Figure 4.

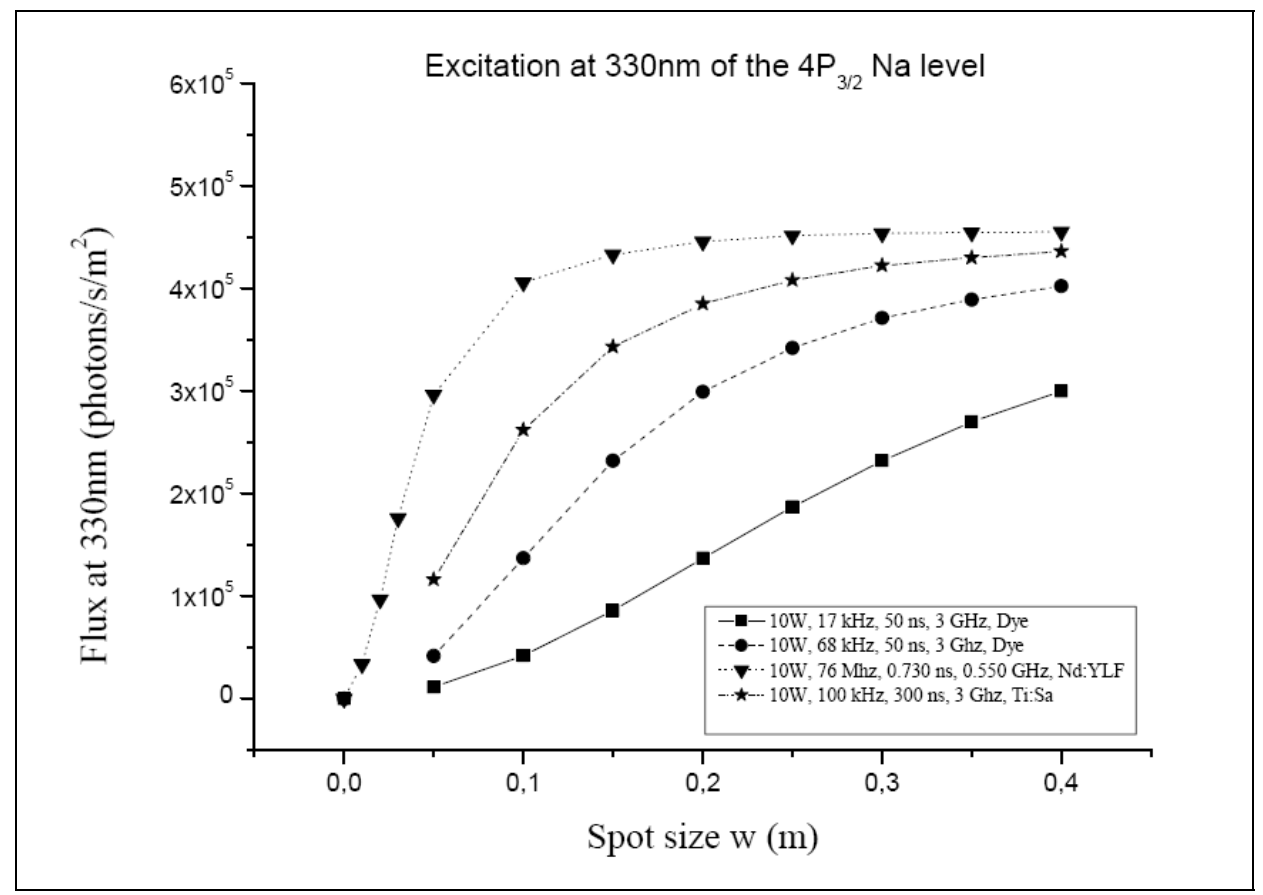

Fig. 4 The flux at 330nm from a modeless $330 \mathrm{~nm}$ laser guide star delivering $10 \mathrm{~W}$ in the mesosphere as a function of the spot size. The flux at $589 \mathrm{~nm}$ is twice as large as the flux at $330 \mathrm{~nm}$. The sodium column density is assumed to be $4 \times 10^{9}$ atom $/ \mathrm{cm}^{2}$.

If a sodium column density of $4 \times 10^{9}$ atoms $/ \mathrm{cm}^{2}$ is considered as a good average, seasonal variations can bring it down to half this value. Further studies should therefore divide by a factor two the values given on Figure 4 to be more conservative, but we have used so far the values given on the above curves. We currently envision using half the $589 \mathrm{~nm}$ return flux for the polychromatic LGS and the other half for the wave front sensor of Pueo Hou as for a traditional laserbased AO system.

\subsection{Launching the laser}

The PLGS tilt determination is based on the measurement of the variations of the relative position of the 330nm and the $598 \mathrm{~nm}$ guide stars. The accuracy of this measurement is a function of the number of photons available on the image of each of its components and of the size of the images themselves. In the ELP-OA simulations, the launch telescope is supposed to work with an AO-corrected beam on the uplink. Is there enough flux back in a $50 \mathrm{~cm}$ telescope to actually make even a low order AO system work? A first round of simulations has been made with the following assumptions:

Taking into account the transmission of the atmosphere on Mauna Kea on the downlink, the $4 \times 10^{5}$ photons $/ \mathrm{s} / \mathrm{m}^{2}$ from $10 \mathrm{~W}$ at the level of the mesosphere translate to 2 photons $/ \mathrm{s} / \mathrm{cm}^{2} / \mathrm{W}$ at the entrance pupil of the telescope. Assuming a transmission (including the quantum efficiency of the detector) of the AO system on the launch telescope of 50\%, and a read noise of 2 electrons, two AO systems were simulated: a $3 \times 3$ and a $4 \times 4$ Shack-Hartman and the short-exposure Strehl ratio computed for different laser powers as a function of the sampling frequency. Correcting only the tip-tilt does not improve anything, as only short exposures are considered, and the corresponding Strehl ratio is $51 \%$. The corresponding curves are shown on Figure 5. 


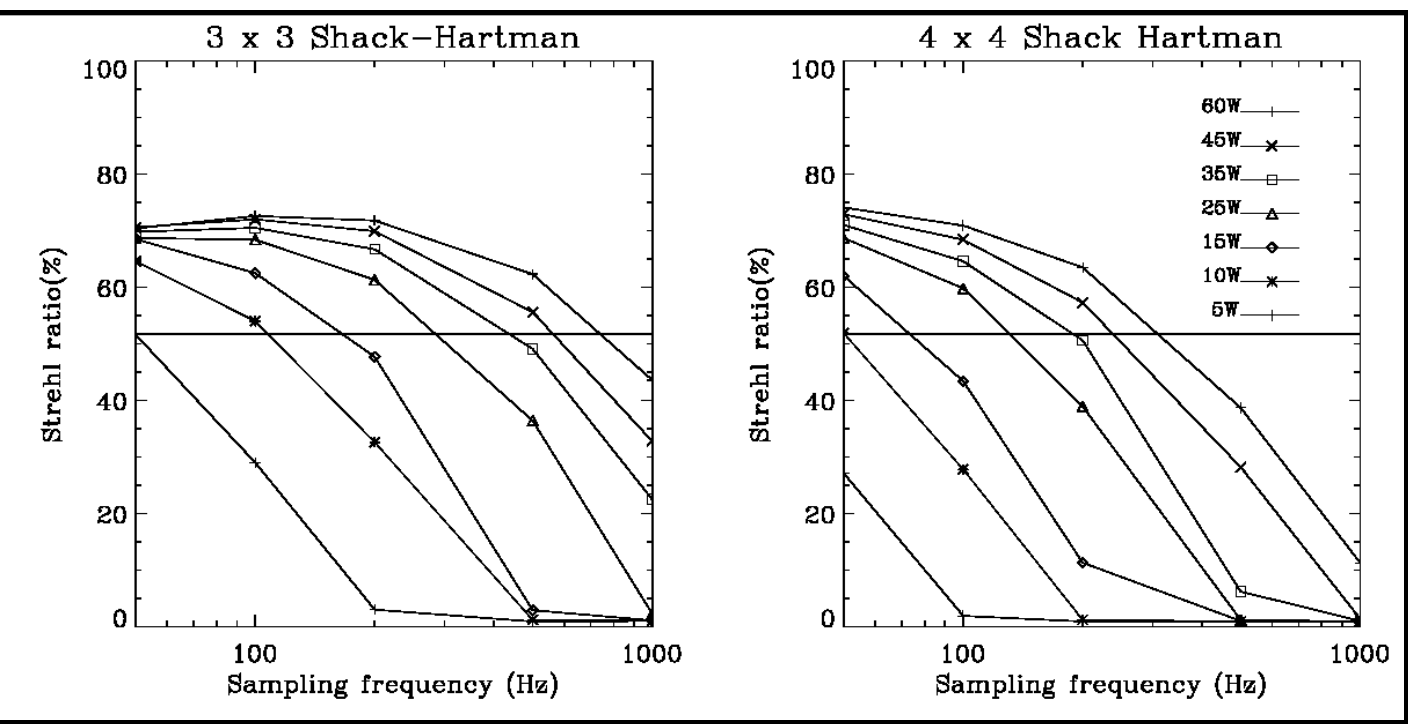

Fig. 5. Short exposure Strehl ratio on the LGS for an AO corrected beam as a function of the sampling rate for various powers of the $330 \mathrm{~nm}$ laser at the level of the mesosphere. Flux values are scaled from a flux of $4 \times 10^{5}$ photons $/ \mathrm{s} / \mathrm{m}^{2}$ from $10 \mathrm{~W}$ of power at the mesosphere (see Fig. 4). Horizontal line at $51 \%$ is the average uncorrected short exposure Strehl ratio.

The launch telescope diameter is optimized to provide the best possible uncorrected short exposure images $\left(\sim 3 \times \mathrm{r}_{0}\right)$. A low order adaptive optics can be used to improve the image quality, but care should be exercised because photon noise introduced in the loop can degrade the correction to below natural levels. A 4x4 Shack Hartman is simply too photon starved to provide any such improvements, while a $3 \times 3$ will indeed improve the Strehl ratio of the PLGS, providing around $65 \%$. More power can help, but there is no way to do better that around $75 \%$.

\subsection{Simulating Pueo Hou and looking at the VASAO science}

Simulations were made using the Yao code written by F. Rigaut using the Yorick language, with 3000 Monte Carlo iterations. On the Pueo Hou side, the telescope is $3.6-\mathrm{m}$ in diameter with a central obstruction; the launch telescope is $50 \mathrm{~cm}$ in diameter.

The atmosphere has an $\mathrm{r}_{0}$ of $16 \mathrm{~cm} . \mathrm{D} / \mathrm{r}_{0}$ is therefore around 20 for the $3.6-\mathrm{m}$ telescope and 3 for the launch telescope. Wind speeds and layers are set as following: $40 \%$ on the ground $(\mathrm{v}=11 \mathrm{~m} / \mathrm{s}), 20 \%$ at $400 \mathrm{~m}(\mathrm{v}=20 \mathrm{~m} / \mathrm{s}), 30 \%$ a $6 \mathrm{~km}$ $(\mathrm{v}=29 \mathrm{~m} / \mathrm{s})$, and the remaining $10 \%$ at $9 \mathrm{~km}(\mathrm{v}=35 \mathrm{~m} / \mathrm{s})$.

As a first step, the deformable mirror is analog to the one used for the AO component of $\mathrm{NICI}^{21}$, modified to match CFHT's pupil. Some of its 85 electrodes were regrouped to improve performances and reduce degrees of freedom, ending with 72 electrodes.

Figures 6 shows the corresponding simulated performances of Pueo Hou at various wavelengths, from 0.5 to $2.5 \mu \mathrm{m}$, as a function of the distance from the guide star. At this stage, none of the photon noise or detector noise is taken into account. The error on the tilt measurements from the PLGS and V2S are not taking into account either: they should however be small enough to have only limited effect on the degradation of the images. determination so results are clearly on the optimistic side.

VASAO is clearly not a wide-field instrument! In the visible, while the FWHM of the images can be 50mas or better in $\mathrm{R}$ or below, the field is around 15 arc-second in diameter. Therefore, the scientific applications in the visible will be mainly targeted to single objects or dense clusters. Moreover, with the relatively small size of the telescope, very low surface brightness object will not be easy targets, while the gain in resolution and exposure time will be important on point-like sources. A first pass on simulations of the focal plane sensitivity gives, in a hour with signal to noise ration of five, the following limiting magnitudes: $g=27.4, \quad r^{\prime}=27.5, i^{\prime}=27.1$ and $z^{\prime}=26.4$ These numbers will obviously be better determined as the performances of the overall system is better known. The VASAO science case is now taking shape in parallel with the feasibility study. Integral field spectroscopy in the visible at high spatial resolution is still missing and could make good use of VASAO. The imaging capabilities will complement, and even improve at low 
wavelength, the resolution of HST images (or perhaps replace it if HST is not available in a few years from now). The study of binary systems or strong lenses would definitely benefit from such a high resolution. Finally, observing in the infrared will still be interesting for imaging areas where no sources are available for natural tilt correction on larger telescopes: coronography or environment of sources with a complex core, accretion disks around young embedded stars...
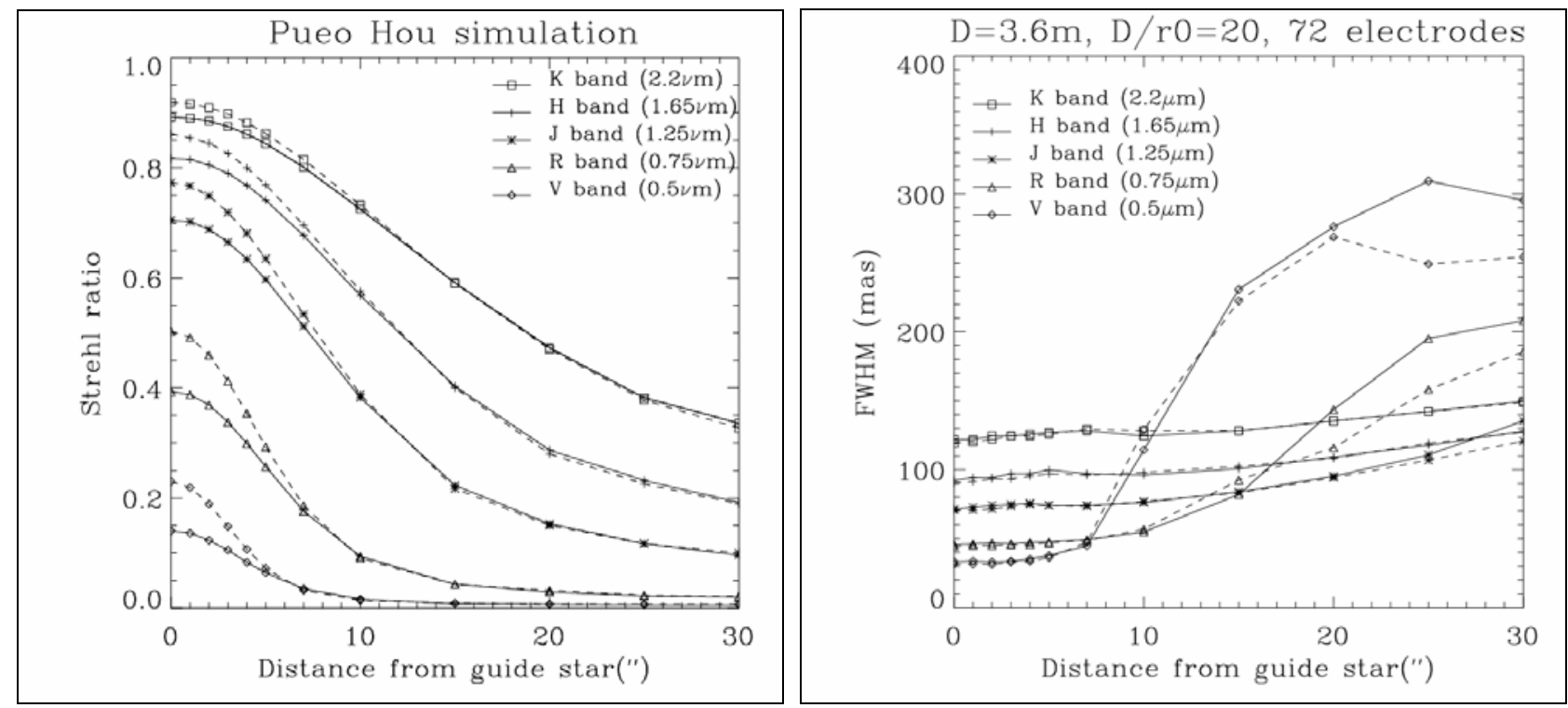

Fig. 6. Pueo Hou simulation: On the left, the Strehl ratio and, on the right, the FWHM in milliarcseconds as a function of the distance from the laser guide star. The dotted lines correspond to the results obtained with a natural guide star, while the solid lines show the LGS results. No noise (photon or readout) is taken into account in this simulation, and tilt correction from the PLGS is assumed to be perfect, so results are clearly optimistic.

\section{CONCLUSION: THE CHALLENGES}

In the middle of a feasibility study, it is obviously difficult to draw definite conclusions. However, enough work has been done to show some of the areas where difficulties will arise:

- Every key system (AO on the launch telescope, polychromatic tilt measurement, Pueo-Hou) will be photon-starved. It is too early in the study to have a clear picture of the photon flux really available on each of these components. The use of a single laser at $330 \mathrm{~nm}$ for both PLGS generation and AO correction is very appealing, but a clear assessment of the photon flux needed is required to evaluate the feasibility of this approach.

- The PLGS will have to be made small to give the accuracy needed on the position of its two components. It will actually need to be smaller than achieved by anybody so far, and by using the LGS itself to correct the laser on tis way up.

- Some of the optical components will be difficult to fabricate if we want to maintain the optical layout of Pueo when moving to Pueo Hou. The wave front sensor pick-up mirror will have to take around half the flux at 589nm and let everything else pass. T2S will have to get all the $330 \mathrm{~nm}$ and $589 \mathrm{~nm}$ photons from the PLGS, and let everything else go to the science field, ideally from $350 \mathrm{~nm}$ to the near infrared.

- Vibration sensing and atmospheric tilt measurements will have to be properly combined to allow a good "blind" fast tracking of the science object, while low frequency guiding will be insured by the telescope Cassegrain bonnette guiding (as done in the current configuration of Pueo).

These difficulties raise many questions about the real feasibility of a whole sky visible AO system, questions which should be answered in the years to come. The reader can follow the progress of VASAO by regularly visiting the VASAO web page ${ }^{22}$. 


\section{REFERENCES}

1. R.D. McClure et al "An Image Stabilization High-Resolution Camera for the Canada-France-Hawaii Telescope" PASP 101, 1156 (1989).

2. F. Roddier "Curvature sensing and compensation: a new concept in adaptive optics". Appl. Optics, 27, 1223-1225 (1988).

3. R. Arsenault et al "PUEO: the Canada-France-Hawaii Telescope adaptive optics bonnette I: system description" Proc. SPIE Vol. 2201, p. 833-842, Adaptive Optics in Astronomy, Mark A. Ealey; Fritz Merkle; Eds. (1994).

4. http://www.cfht.hawaii.edu/Instruments/Imaging/AOB/

5. F. Rigaut et al "Performance of the Canada-France-Hawaii Telescope Adaptive Optics Bonnette" PASP, 110, Issue 744, 152-164 (1998).

6. http://www.cfht.hawaii.edu/Instruments/Imaging/CFH12K/

7. http://www.cfht.hawaii.edu/Instruments/Imaging/MegaPrime/

8. http://www.cfht.hawaii.edu/Instruments/Imaging/WIRCam/

9. R. Foy and A. Labeyrie "Feasibility of adaptive telescope with laser probe" Astron. Astrophys. 152, L29-32 (1985).

10. http://www2.keck.hawaii.edu/optics/lgsao/

11. R. Foy et al. "The polychromatic artificial sodium star: a new concept for correcting the atmospheric tilt". Astron. Astrophys. Supp., 111, 569 (1995).

12. R. Foy et al. "ELPOA: toward the tilt measurement from a polychromatic laser guide star". Proc. SPIE 4007, 284295, Adaptive Optical Systems Technology, Peter L. Wizinowich; Ed. (2000).

13. R. Foy et al. "Feasibility study of the polychromatic laser guide star". Adaptive Optical System Technologies II. Edited by Wizinowich, Peter L.; Bonaccini, Domenico. Proceedings of the SPIE, 4839, 484-491 (2004).

14. M. Schõck et al. "Performance analysis of polychromatic laser guide stars used for wavefront tilt sensing". MNRAS, 337, Issue 3, 910-920 (2002).

15. A. Tokovinin. "Pendular seismometer for correcting telescope vibrations" MNRAS 316, Issue 3, 637-641 (2000).

16. M. Schoeck "PASS-2: quantitative photometric measurements of the polychromatic laser guide star". Proc. SPIE Vol. 4007, p. 296-307, Adaptive Optical Systems Technology, Peter L. Wizinowich; Ed. (2000).

17. J. Girard "Validation sur le ciel du concept d'étoile laser polychromatiques" Doctoral Dissertation- Université Claude Bernard - Lyon 1 (2005).

18. J.P. Pique and S. Farinotti "Efficient modeless laser for a mesospheric laser guide star" JOSA B 20, 2093 (2003).

19. K. Ho et al "Update report on FlyEyes: a dual CCD detector system upgrade for PUEO". Optical and Infrared Detectors for Astronomy. Edited by James D. Garnett and James W. Beletic. Proceedings of the SPIE, Volume 5499, pp. 395-405 (2004).

20. J.P. Pique "Polychromatic laser guide star using a single laser at $330 \mathrm{~nm}$ " These Proceeedings.

21. http://www.gemini.edu/sciops/instruments/nici/niciIndex.html

22. http://www.cfht.hawaii.edu/VASAO/ 